\title{
Correlating the force network evolution and dynamics in slider experiments
}

\author{
Chao Cheng ${ }^{1, *}$, Aghil Abed Zadeh ${ }^{2, * *}$, and Lou Kondic ${ }^{1, * * *}$ \\ ${ }^{1}$ Department of Mathematical Sciences, New Jersey Institute of Technology, Newark, NJ, 07102 \\ ${ }^{2}$ Department of Physics and Department of Neurobiology, Duke University, Durham, NC, 27708
}

\begin{abstract}
The experiments involving a slider moving on top of granular media consisting of photoelastic particles in two dimensions have uncovered elaborate dynamics that may vary from continuous motion to crackling, periodic motion, and stick-slip type of behavior. We establish that there is a clear correlation between the slider dynamics and the response of the force network that spontaneously develop in the granular system. This correlation is established by application of the persistence homology that allows for formulation of objective measures for quantification of time-dependent force networks. We find that correlation between the slider dynamics and the force network properties is particularly strong in the dynamical regime characterized by well-defined stick-slip type of dynamics.
\end{abstract}

\section{Introduction}

A wide range of systems exhibit intermittent dynamics as they are slowly loaded, with different dynamical regimes governing many industrial and natural phenomena. In these systems, the energy is loaded gradually with a stable configuration and then is dissipated in fast dynamics with microscopic and macroscopic rearrangements [1]. Examples are fracture [2,3], magnetization [4], and seismic activities $[3,5]$ such as earthquakes, in which the slowly loaded energy relaxes via fast reconfiguration. This intermittent behavior has been observed in a number of granular experiments and simulations [6-9]. In analyzing such behavior, a significant progress has been reached by studying the dynamics of a slider coupled with the boundary of a granular system. A slider can exhibit a wide variety of dynamics, including continuous flows and periodic or intermittent stick-slip behavior [8,10-12].

While a significant amount of research on exploring intermittent dynamics of granular systems has been carried out, not much is known about the connection between particle-scale response and the global dynamics, in particular for experimental systems. In slider experiments $[8,10]$, see also Fig. 1, it is possible to measure particle scale response by using photoelastic techniques. These techniques allow for extracting dynamic information about evolving particle interactions which typically involve meso-scale force networks (so-called 'force chains'). Analysis of such time-dependent weighted networks is not a simple task, and it has evolved through last decades in a variety of different directions, including force network ensemble [13, 14], statistics-based methods $[15,16]$, and network type of analysis $[17,18]$. In

\footnotetext{
*e-mail: cc563@njit.edu

**e-mail: aghil.abed.zadeh@duke.edu

***e-mail: kondic@njit.edu

A video is available at https://doi.org/10.48448/nbkr-pn33
}

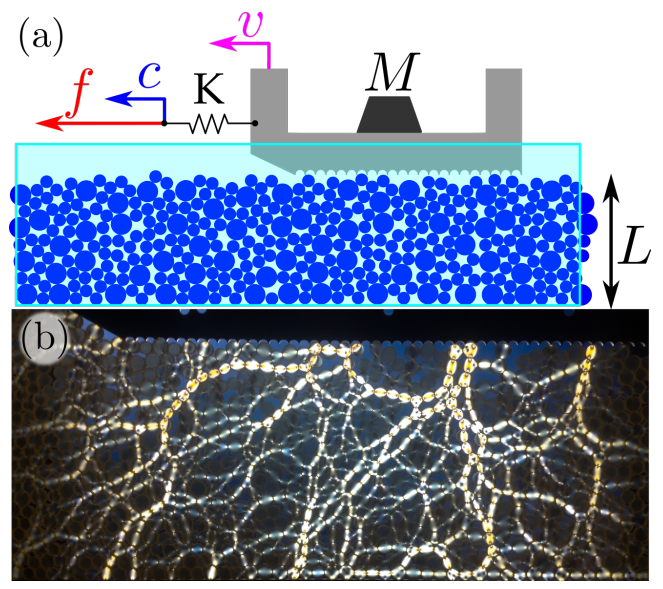

Figure 1: (a) Sketch of the experiment. A slider sits on top of a 2D granular system with photoelastic disks, and is connected to a stage by a spring of constant $k$, pulled by a constant speed $c$. A force gouge measures the force $f$; the granular medium is imaged with fast cameras. (b) Photoelastic response during loading [10]. Reprinted with permission from [10].

the present work, we will consider application of persistent homology $(\mathrm{PH})$, which allows for formulating precise and objective measures of static and dynamic properties of the force networks. This approach has been used extensively in analysis of the data obtained via discrete element simulations in the context of dry granular matter $[19,20]$ and suspensions [21], but its application to experimental data has so far been rather sparse $[22,23]$. We show that this method allows to develop clear correlations between the static and dynamic properties of the force networks on micro- and meso-scale and the macro-scale system dynamics. 


\section{Techniques}

\subsection{Experimental techniques}

In our experiments, as shown in Fig. 1(a), a stage pulls a 2D frictional slider with toothed bottom, of a fixed length of $25 \mathrm{~cm}$ and a mass of $M=85 \mathrm{~g}$. The stage, which moves at constant speed $c$, is connected to the slider by a linear spring of stiffness $K$. The slider rests on a vertical bed of fixed depth $L=9.5 \mathrm{~cm}$ and length $1.5 \mathrm{~m}$, consisting of bi-disperse photo-elastic disks with radii of $0.4 \mathrm{~cm}$ and $0.5 \mathrm{~cm}$. A camera, recording the photo-elastic response of the medium at $120 \mathrm{fps}$, is connected to the stage. We also record the force $f$ experienced by the spring.

We consider three experiments characterized by different configurations of $c$ and $K$ : Exp. $1: K=14 \mathrm{~N} / \mathrm{m}$, $c=0.5 \mathrm{~mm} / \mathrm{s}$; Exp. 2: $K=70 \mathrm{~N} / \mathrm{m}, c=0.5 \mathrm{~mm} / \mathrm{s}$, and Exp. 3: $K=70 \mathrm{~N} / \mathrm{m}, c=1.0 \mathrm{~mm} / \mathrm{s}$. The total number of analyzed frames (images) is 30,000 for each experiment, corresponding to 250 seconds of physical time.

\subsection{Image processing}

The goal of the image processing in this study is to reveal clear force signal and reduce noise effects as much as possible. As the fast imaging in our experiments constrains the resolution of images, we use brightness method to capture force information, which works better that $G^{2}$ method for the type of data collected, see [24]; similar approach was used in [23]. We first remove background noise from the original images by applying a filter that removes pixels of brightness below chosen threshold value so to remove low light area and particle textures. Multiple threshold values were investigated, giving no quantitatively difference in the results of the topological analysis that follow; we typically use threshold value of 90 (the maximum brightness is 255), which is appropriate for capturing the relevant information. After thresholding, the image brightness is linearly mapped to 0-255 range. MATLAB builtin functions imerode and imdilate were applied to slightly dilate the bright regions so to fill the gaps between neighboring particles where in force chains are connected, and then to erode away the unwanted excessive dilation to restore the force networks with more accuracy. Fig. 2 shows an example of image processing; in our computations discussed in what follows, we use grey scale version of the figures such as Fig. 2 for the purpose of computing considered topological measures.

\subsection{Topological measures}

Persistence homology (PH) allows for formulating objective measures describing force networks in both simulations and experiments. Analysis of experimental data, such as the ones considered here, presents some challenges which are discussed in some detail in [20].

Each experimental image can be considered as an array of pixel brightness $\theta \in[0,255]$. Since the pixels of high brightness correspond to the particles experiencing large forces, we can apply $\mathrm{PH}$ to the pixels to track and quantify their connectivity to extract (approximate)
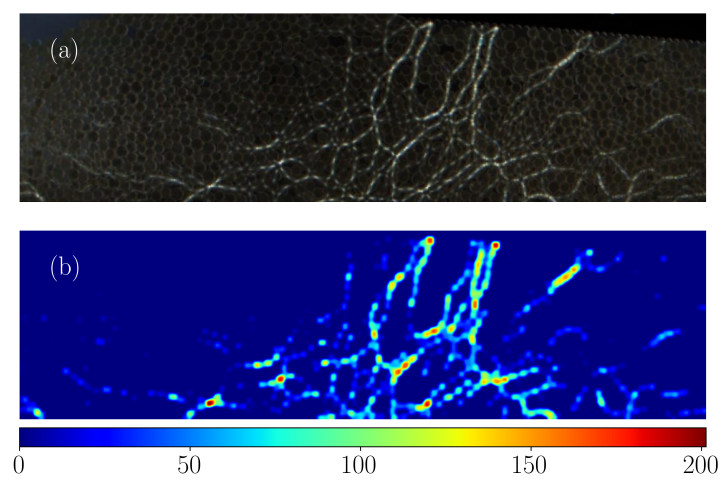

Figure 2: An example of image processing. (a) An original experimental image. (b) A processed image; color scheme shows pixel intensity.

information about the actual force networks. PH techniques essentially encode the appearance (birth) and disappearance (death) of force networks by generating persistence diagrams (PDs) that encode the birth and death brightness levels for components (loosely speaking, force chains), and loops (cycles). The PDs therefore reduce the complexity of underlying force networks into point clouds where the coordinates are $\left(\theta_{\text {birth }}, \theta_{\text {death }}\right)$ and each point represents an object that could be either connected component (chain) or a loop (cycle). The lifespan of an object is defined as $\theta_{\text {birth }}-\theta_{\text {death }}$, measuring how long the object lasts as the threshold is varied. Total persistence (TP) of a PD is defined as the sum of all lifespan of the points, $\mathrm{TP}(\mathrm{PD})=\sum_{\left(\theta_{\text {birth }}, \theta_{\text {death }}\right) \in P D}\left(\theta_{\text {birth }}-\theta_{\text {death }}\right)$, which further reduce the complexity of force networks to a single number [25]. Note that TP is influenced by both how many components there are, and by their lifespans.

Another quantity related to PDs is the distance (or difference) between them. The distance measures essentially the cost of mapping points in one PD to those in another $\mathrm{PD}$; in the case of different number of points, the extra ones are mapped to the diagonal. In particular, the degree$q$ Wasserstein distance between two persistence diagrams PD and PD' is defined as

$$
d_{W q}\left(P D, P D^{\prime}\right)=\inf _{\gamma: \mathrm{PD} \rightarrow \mathrm{PD}^{\prime}}\left(\sum_{p \in \mathrm{PD}}\|p-\gamma(p)\|_{\infty}^{q}\right)^{1 / q},
$$

where $\gamma$ is a bijection between points from PD to PD', $\gamma: \mathrm{PD} \rightarrow \mathrm{PD}^{\prime}$. In the present work we use $q=2$ and carry out the calculations using the method discussed in [26, 27].

\section{Results}

\subsection{Structural response}

Figure 3 shows the calculated velocity of the slider and the measured force, $f$, on the spring. This figure illustrates clearly the slider's dynamics. We note that Exp. 1 exhibits crackling stick-slip behavior as the driving rate is small. During a stick, the spring builds up the stress, while the slider is almost fixed, until the spring eventually yields, leading to a sharp velocity jump and drop of the force. 


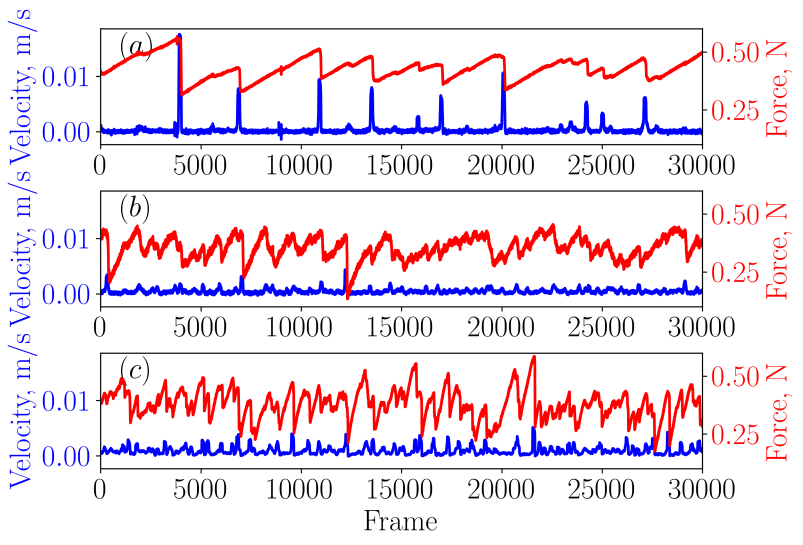

Figure 3: Sliders' velocity and spring force for the considered experiments: (a) Exp. 1, (b) Exp. 2, (c) Exp. 3.

The system behaves more similarly to a continuous flow for Exps. 2 and 3, as also discussed in [10].
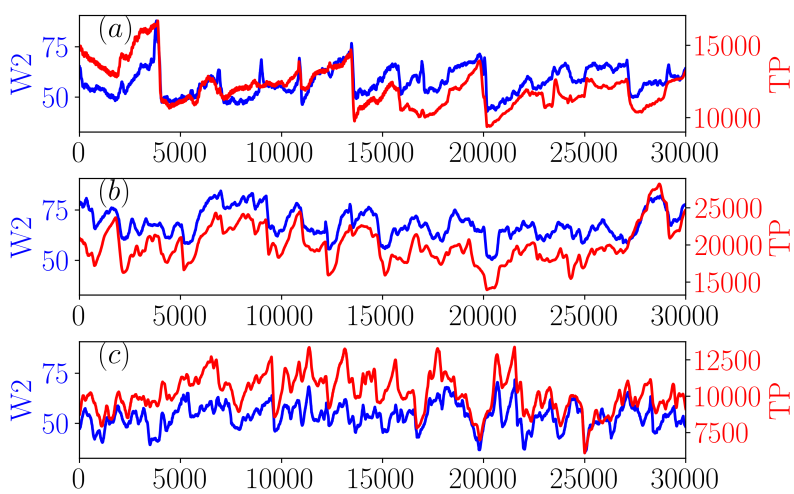

Figure 4: $W 2$ distance and total persistence, corresponding to the experiments shown in Fig. 3.

Next, we proceed with considering persistence measures. Figure 4 shows the Wassertein $q=2$ distance (W2) for the considered experiments, as well as the total persistence (TP), for the same time interval as shown in Fig. 3. Direct comparison with Fig. 3 shows good agreement between the force network measures, W2 and TP, from the one side, and the sliders' velocity and the spring force, on the other. Figure 5 illustrates in more detail the degree of agreement between the force and W2 for Exp. 1. We note that this experiments shows particularly good agreement, suggesting stronger correlation between the force on the slider and force network properties for well defined stickslip dynamics. We note good agreement between W2 and $\mathrm{TP}$, suggesting the existence of a correlation between the 'strength' of the network measured by TP, and its temporal evolution, measured by W2.

Having established correspondence between the slider dynamics and the force network, we proceed to discuss whether such correlation could be explored for predictive purposes. To explore this question, we consider the cross-correlation between considered quantities. More precisely, consider two time series $x_{t}, y_{t}$, with the

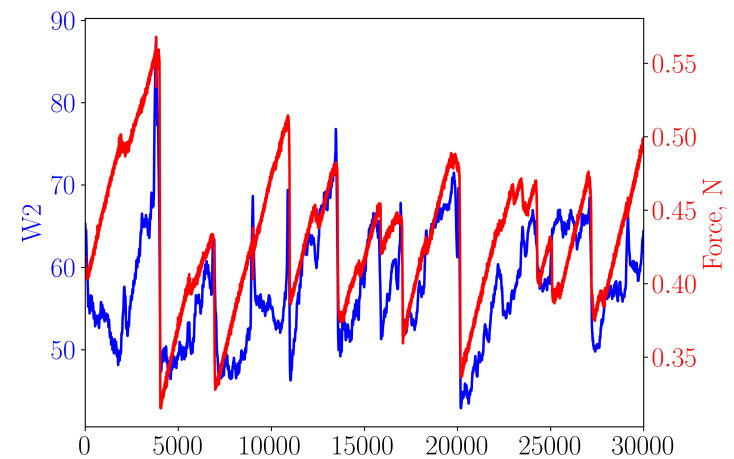

Figure 5: Force on the slider and W2 distance (data from Fig. 3a) and 4a)).

data $\left(x_{1}, x_{2}, \cdots, x_{m}\right)$, and $\left(y_{1}, y_{2}, \cdots, y_{m}\right)$. The crosscovariance is defined by

$$
c_{x y}(k)=\frac{1}{m} \sum_{t=1}^{m}\left(x_{t}-\bar{x}\right)\left(y_{t-k}-\bar{y}\right)
$$

where $\bar{x}=\sum_{i=1}^{m} x_{i} / m, \bar{y}=\sum_{i=1}^{m} y_{i} / m$, and $k=$ $0, \pm 1, \pm 2, \cdots$ is the chosen lag. When $m$ is outside the range of $y, y_{m}=0$. Note that for the positive lag $k, x_{t}$ is correlating with $y_{t-k}$ (at earlier time), which means that we may be able to use such correlation to predict the future $x$ from earlier $y$. Finally, we define the sample standard deviations of the series as

- $s_{x}=\sqrt{c_{x x}(0)}$, where $c_{x x}(0)=\operatorname{Var}(x)$.

- $s_{y}=\sqrt{c_{y y}(0)}$, where $c_{y y}(0)=\operatorname{Var}(y)$.

The cross-correlation coefficient is given by

$$
r_{x y}(k)=\frac{c_{x y}(k)}{s_{x} s_{y}}, k=0, \pm 1, \pm 2, \cdots
$$

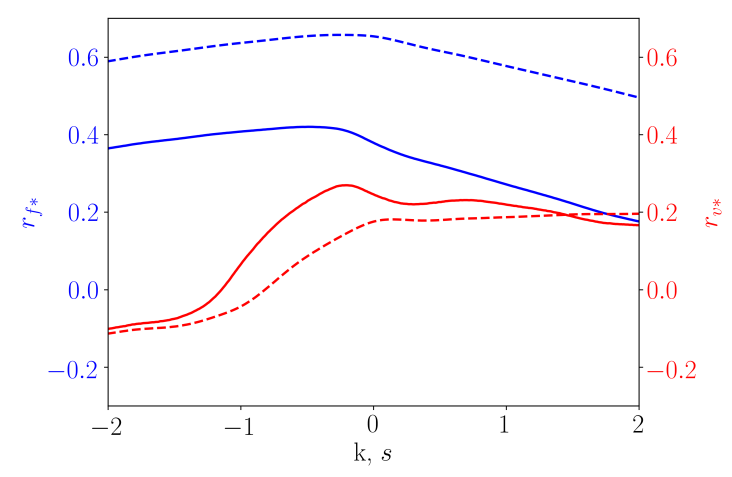

Figure 6: Cross correlation coefficient as a function of lag $k$ (in seconds). $r_{f *}$ and $r_{v *}$ for Exp. 1, where $*$ stands either for W2 distance (solid line), or for TP (dashed line).

Figure 6 shows the cross-correlation coefficients for Exp. 1, where $r_{f *}, r_{v *}$ correspond to the cross-correlation coefficient between the $f$ or $v$ and the measure of interest *, which can be $w$ for W2 distance or $t$ for TP. Focusing first at the results without lag, $k=0$, we note that the $r_{f *}$ 
correlations are higher than the $r_{v *}$ ones; we expect that this is due to the fact that the velocity data were obtained by taking a discrete derivative of the slider position data, introducing further noise which may blur the actual signal. The $r_{f *}$ results show that the correlation is higher for the TP data, which is not surprising since TP is expected to reflect the force on the slider, while W2 distance measures the temporal difference in PDs.

Considering next the results for non-zero lags, we note a different behavior of $r_{f *}$ and $r_{v *}$ curves, with $r_{v *}$ curves rising from negative to positive as lag is increased. Such difference results from the fact that the structure of the force and velocity profiles are rather different. The velocity profile shows sharp transitions, while the force profile slowly builds up during the stick periods and drop dramatically at the events. More importantly, we note that within a reasonable range of positive lags, the $r_{f w}$ and $r_{f t}$ are still significant in size, suggesting a potential for predictability. It should be noted however, that since the main part of the data is in a "stick" region, the correlation will naturally weight more on these data points. A more insightful procedure would involve correlating the measures just before a slip event. Such an analysis would however require more data points, and possibly also more detailed experimental input and therefore we leave if for the future work.

Before closing, we note that for Exp. 2 and 3 we obtain consistent results, however the correlations are weaker; e.g., the max of $r_{f t}$ goes down from 0.65 (Exp. 1), to 0.45 (Exp. 2), and to 0.3 (Exp. 3). Despite the fact that all three experiments fall into the same category of stick-slip in the dynamic phase diagram [10], clearly (see Fig. 3) the slip events are much stronger and better defined for Exp. 1, suggesting that in particular for such situation the persistence measures provide insightful information.

\section{Conclusion}

We find that the tools of persistent homology (PH) allow for correlating the dynamics of a slider and the photoelastic response of granular particles. In particular stick and slip regimes of the slider dynamics are well captured by the PH measures. These results suggest that there is a potential for developing predictive capabilities by analyzing the response of the force network to an external perturbation. One open question is how precise should the information about the forces between the granular particles be so to allow for further development of this potential. We hope that our results set up a stage for this future work.

\section{Acknowledgements}

The authors acknowledge many insightful conversations with J. Barés, R. Basak, (late) R. Behringer, M. Carlevaro, K. Daniels, M. Kramar, K. Mischaikow, J. Morris, L. Pugnaloni, A. Singh, J. Socolar, and H. Zheng. $\mathrm{CC}$ and LK acknowledge support by the ARO grant No. W911NF1810184.

\section{References}

[1] J.P. Sethna, K.A. Dahmen, C.R. Myers, Nature 410, $242(2001)$
[2] J. Barés, M.L. Hattali, D. Dalmas, D. Bonamy, Phys. Rev. Lett. 113, 264301 (2014)

[3] J. Barés, A. Dubois, L. Hattali, D. Dalmas, D. Bonamy, Nature Comm. 9, 1253 (2018)

[4] J.S. Urbach, R.C. Madison, J.T. Markert, Phys. Rev. Lett. 75, 276 (1995)

[5] J. Davidsen, G. Kwiatek, Phys. Rev. Lett. 110, 068501 (2013)

[6] D.V. Denisov, K.A. Lőrincz, W.J. Wright, T.C. Hufnagel, A. Nawano, X. Gu, J.T. Uhl, K.A. Dahmen, P. Schall, Sci. Rep. 7, 43376 (2017)

[7] C. Liu, E.E. Ferrero, F. Puosi, J.L. Barrat, K. Martens, Phys. Rev. Lett. 116, 065501 (2016)

[8] A.A. Zadeh, J. Barés, J.E.S. Socolar, R.P. Behringer, Phys. Rev. E 99, 052902 (2019)

[9] K.A. Murphy, K.A. Dahmen, H.M. Jaeger, Phys. Rev. X 9, 011014 (2019)

[10] A.A. Zadeh, J. Barés, R.P. Behringer, Phys. Rev. E 99, 040901 (2019)

[11] M. Pica Ciamarra, E. Lippiello, C. Godano, L. de Arcangelis, Phys. Rev. Lett. 104, 238001 (2010)

[12] M. Pica Ciamarra, A. Coniglio, Phys. Rev. Lett. 103, 235701 (2009)

[13] B.P. Tighe, J.H. Snoeijer, T.J.H. Vlugt, M. van Hecke, Soft Matter 6, 2908 (2010)

[14] S. Sarkar, D. Bi, J. Zhang, R.P. Behringer, B. Chakraborty, Phys. Rev. Lett. 111, 068301 (2013)

[15] J. Peters, M. Muthuswamy, J. Wibowo, A. Tordesillas, Phys. Rev. E 72, 041307 (2005)

[16] L. Bo, R. Mari, C. Song, H.A. Makse, Soft Matter 10, 7379 (2014)

[17] D.S. Bassett, E.T. Owens, K.E. Daniels, M.A. Porter, Phys. Rev. E 86, 041306 (2012)

[18] D. Walker, A. Tordesillas, Phys. Rev. E 85, 011304 (2012)

[19] S. Ardanza-Trevijano, I. Zuriguel, R. Arévalo, D. Maza, Phys. Rev. E 89, 052212 (2014)

[20] M. Kramár, A. Goullet, L. Kondic, K. Mischaikow, Physica D. 283, 37 (2014)

[21] M. Gameiro, A. Singh, L. Kondic, K. Mischaikow, J.F. Morris, Phys. Rev. Fluids 5, 034307 (2020)

[22] J.A. Dijksman, L. Kovalcinova, J. Ren, R.P. Behringer, M. Kramár, K. Mischaikow, L. Kondic, Phys. Rev. E 97, 042903 (2018)

[23] T. Takahashi, A.H. Clark, T. Majmudar, L. Kondic, Phys. Rev. E 97, 012906 (2018)

[24] A.A. Zadeh, J. Barés, T.A. Brzinski, K.E. Daniels, J. Dijksman, N. Docquier, H.O. Everitt, J.E. Kollmer, O. Lantsoght, D. Wang et al., Gran. Matt. 21 (2019)

[25] L. Kondic, M. Kramár, L.A. Pugnaloni, C.M. Carlevaro, K. Mischaikow, Phys. Rev. E 93, 062903 (2016)

[26] A data exploration tool for TDA, https://github.com/ rachellevanger/tda-persistence-explorer

[27] Statistical tools for TDA, https://cran.r-project.org/ web/packages/TDA/index.html 